\title{
Energy Management and Health: Implication of Nervous System Superconductor-Like Behaviour
}

\author{
Hafedh Abdelmelek $^{1 *}$, Amel Hanini ${ }^{1}$, Linda Saili1,2, Amina Azzouz ${ }^{1,2}$, Ezzedine Nebil ${ }^{3}$, Zihad Bouslama², Mohsen Sakly ${ }^{1}$ and Mbainaibeye \\ Jérôme ${ }^{1}$ \\ ${ }^{1}$ Laboratoire de Physiologie Intégrée, Faculté des Sciences, Université de Carthage, Jarzouna, Bizerte 7021, Tunisie \\ 'Laboratoire d'Ecologie des Systèmes Terrestres et Aquatiques, Faculté des Sciences, Université Badji Mokhtar, BP 12, 23000 Sidi Amar, Annaba, Algérie \\ ${ }^{3}$ Clinique d'Acupuncture de Bizerte, 7000 Bizerte, Tunisie
}

\begin{abstract}
Physiological studies have implicated different mediator in energy management in nervous system and body metabolic rate. Health result in a good balance between positive $(+1)$ or negative $(-1)$ energy leading to physiological energy (E0). Interestingly, diseases could be explained by an unbalance of energy regulation of nervous system (alteration of superconductor-like behaviour by environment factors) and/or body metabolic rate. Recent years several studies investigating the implication of electrical signals at very low temperature in animal species adaptation and life. In this paper, the electric resistivity $(R)$ at different temperatures $(T)$ between 300 to $200 \mathrm{~K}$ in frog sciatic nerves was studied. When the electrical contacts were leaned into sciatic nerve of the four studied species a striking decrease of nerve resistivity was observed (frog $252 \mathrm{~K}$ ); showing a superconductor-like behavior that could be associated to good health related to good emotions and metabolic equilibrium in Human and animals. Analysis of electrical properties demonstrates clearly that alteration of superconductor-like behaviour by environmental factors point to the development metabolic disorders and diseases.
\end{abstract}

Keywords: Energy; Management; Time; Health; Superconductorlike behaviour

\section{Introduction}

Physiological studies have shown that different kind of mediator implicated in energy management explain the balance between health and disease. Abdelmelek et al. [1] reported a direct action of glucagon on avian thermoregulatory mechanisms. Interestingly, cold and glucagon induced an increase of metabolic rate associated to the mobilization of lipids and sympatho-adrenal stimulation. The changes in peripheral noradrenergic activity during cold acclimation could be associated with adaptive changes leading to non-shivering thermogenesis. We have à balance resulting from the nervous system and body interactions. Moreover, the effect of cold acclimatization on the regional distribution of monoamines in duckling spinal cord was studied. In thermoneutral controls (TN), the high dopamine (DA) to norepinephrine (NE) ratios ( 0.25 at 4 weeks of age and 0.15 at 6 weeks of age) suggest the presence of specific (non precursor) dopaminergic pools in cervical spinal cord. DA levels and the ratio of DA to NE were lowered by age and cold exposure in the cervical cord. In TN ducklings, serotonin (5-HT) and its metabolite 5-hydroxyindoleacetic acid (5HIAA) showed a decline with age in the spinal cord, indicating that this system is affected with development, whereas cold exposure prevents this decrease. The contents of 5-HT (+58\%), 5-HIAA (+93\%) and the ratio of 5-HIAA to 5 -HT $(+50 \%)$ are higher in the cervical spinal cord of cold acclimated than in TN ducklings. These results indicate that central monoaminergic systems are markedly affected by age and cold exposure [1]. Moreover, physiological study have shown neurochemical and electrical changes in nervous system during temperature variations could be associated with adaptative changes leading to evolution of species from poikilotherm to endotherm as well know model for energy management [1-3]. Besides the action of monoamines other electrical properties such as action potential or superconductorlike behavior play a key role in the optimization of nerve network and energy management. Previous study suggested the presence of adaptative mechanisms involving the spinal cord and the sciatic nerve dopaminergic and serotonergic pathways during cold acclimation $[1,3]$.
Recent studies reported an increase in myelin sheaths during evolution that could be implicated to the neuromodulation of two components: the neurochemical transmission at low temperature and the adaptative nervous system response to different environmental stimuli giving to animal the so called good health. Previous study by Mbainaibeye et al. [4-8] revealed different electrical responses of frog sciatic nerves during the decrease of temperature. It might therefore be expected that natural selection on neuron or nerve conduction could have caused several structural and functional changes $[1,4,5]$.

The present review report the influence of temperature on the electrical properties of sciatic nerves and to explain the implication of superconductor-like behaviour in health related to energy fluctuation and emotions or health.

\section{Basic Elements of Wavelet Transform}

The basic idea behind the Wavelet Transform is to represent any arbitrary function $f$ as a weighted sum of a set of basis functions $\psi_{s, u}$ which are scaled and shifted version of a single mother wavelet $\psi$. The wavelet decomposition is defined by equation 1 :

$$
\begin{aligned}
& W f(u, s)=\int_{-\infty}^{+\infty} f(t) \frac{1}{\sqrt{s}} \psi\left(\frac{t-u}{s}\right) d t \\
& f(t)=\frac{1}{C_{\psi}} \int_{0}^{+\infty} \int_{-\infty}^{+\infty} W f(u, s) \frac{1}{\sqrt{s}} \psi\left(\frac{t-u}{s}\right) d u \frac{d s}{s^{2}}
\end{aligned}
$$

*Corresponding author: Hafedh Abdelmelek, Laboratoire de Physiologie Intégrée Faculté des Sciences, Université de Carthage, Jarzouna, Bizerte 7021, Tunisie, Tel: (216) 93006057; E-mail: Hafedh.abdelmelek@fsb.rnu.tn

Received September 20, 2017; Accepted September 25, 2017; Published September 29, 2017

Citation: Abdelmelek H, Hanini A, Saili L, Azzouz A, Nebil E, et al. (2017) Energy Management and Health: Implication of Nervous System Superconductor-Like Behaviour. J Health Med Informat 8: 289. doi: 10.4172/2157-7420.1000289

Copyright: ( 2017 Abdelmelek H, et al. This is an open-access article distributed under the terms of the Creative Commons Attribution License, which permits unrestricted use, distribution, and reproduction in any medium, provided the original author and source are credited. 
Citation: Abdelmelek H, Hanini A, Saili L, Azzouz A, Nebil E, et al. (2017) Energy Management and Health: Implication of Nervous System Superconductor-Like Behaviour. J Health Med Informat 8: 289. doi: 10.4172/2157-7420.1000289

Page 2 of 8

Where $\psi_{s, u}(t)=\frac{1}{\sqrt{s}} \psi\left(\frac{t-u}{s}\right)$

There exist special choices of $\psi$ such that the set $\left\{\psi_{s, u}\right\}$ form an orthogonal basis of $L^{2}(R)$. In that case, the wavelet coefficient may be calculated by the equation 3 :

$$
C(s, u)=\left\langle\psi_{s, u}\right\rangle=\int \psi_{s, u}(t) f(t) d t
$$

The wavelet Transform may be considered in its continuous version or in its discrete version depending of the application.

In contrast to Fourier Transform, the Wavelet Transform uses scale and translation as two parameters which allow constructing basis functions to take account of the stationary and the non-stationary behaviour of signal.

The Fourier Transform decomposes the signal using sinus and cosines as basis functions. Equation 4 defines the Fourier Transform of a signal $f(t)$.

$$
\hat{f}(w)=\int_{-\infty}^{+\infty} f(t) e^{-i w t} d t=\int(f(t))(\cos (t)-i \sin (t)) d t
$$

We can see in equation 4 that the signal is globally analyzed and no localized characteristic of the signal may be detected.

One of the solutions to take account of the local characteristics of the signal is to divide the signal using moved window function $g$ to obtain the Short Time Fourier Transform. This transform is defined in equation 5.

$$
\hat{f}(t, w)=\int_{-\infty}^{+\infty} f(s) g(s-t) e^{-i w t} d s
$$

The usual window functions are Hamming, Hanning, Blackman and de Gauss functions. These functions allow calculating ing the temporal resolution and the frequential resolution defined by equations 6 and 7 respectively.

$$
\begin{aligned}
\sigma_{t}^{2} & =\int_{-\infty}^{+\infty} t^{2}|g(t)|^{2} d t \\
\sigma_{f}^{2} & =\int_{-\infty}^{+\infty} w^{2}|\hat{g}(f)|^{2} d f
\end{aligned}
$$

In the case where $|g(t)|=e^{-\frac{t^{2}}{2}}$, then $\sigma_{t}=1$ et $\sigma_{f}=\frac{1}{2 \pi}$. The Figure 1 shows the graphical representation of the time-frequency division using moved window function Figure 1.

In this notion of moved window function or Short Time Fourier

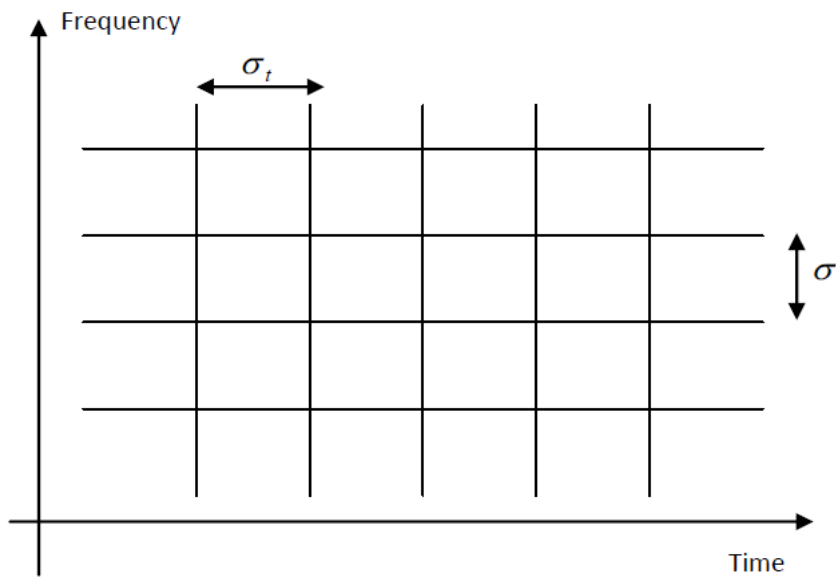

Figure 1: Time-frequency division using moved window function.
Transform, the Gabor Transform also called Gabor atom is defined by equation 8 :

$$
\hat{f}_{G}(t, w)=\frac{1}{\sqrt{2 \pi} \sigma} \int_{-\infty}^{+\infty} f(s) e^{-\frac{(s-t)^{2}}{2 \sigma^{2}}} e^{-i w t} d s
$$

The energy of $g_{u, \xi}$ in concentrated in the neighboring of $u$ in an interval which the width is $\sigma_{t}$. It is easy to verify that the Fourier Transform of $g_{u, \xi}$ can be obtained by the translated version of the Fourier Transform of $g$ where $\xi$ is the translation parameter. This is done by equation 9 :

$$
\hat{g}_{u, \xi}(w)=\hat{g}(w-\xi) e^{-i u(w-\xi)}
$$

The energy of $\xi$ is also in concentrated in the neighboring of $\xi$ in an interval which the width is $\sigma_{f}$.

In the time-frequency representation, the energy of the Gabor atom is represented by the Heisenberg rectangle in the Figure 2.

In contrary to Fourier Transform, analysis with Wavelet Transform allows to vary the temporal and frequential resolution by varying the scale parameter. From the basis function called mother wavelet function $\psi_{u, s}$ it is easy to generate small basis functions $\psi_{u, s}$ defined by equation 10 :

$$
\psi_{u, s}(t)=\frac{1}{\sqrt{s}} \psi\left(\frac{t-u}{s}\right), u \in R, s \succ 0
$$

In equation 10 , the scale parameter $s$ is considered us the inverse of the frequency; so, more small is $s$, more opened is the basis function. In contrary, more big is $s$, less opened is the basis function. Figure 3 shows the time-frequency division using wavelet basis function (we can see the difference compared with the Figure 1 for Short Time Fourier basis function). In Figure 3, the temporal resolution and the frequency resolution are variable depending of scale parameters (Figure 3 ).

Figure 4 presents time-frequency energy of wavelet basis function (we can see the difference compared with Figure 2 for the Gabor atom).

The Figure 4 shows that the time resolution and the frequential resolution may be varying to adapt the signal processing taking account of its stationary or non-stationary properties. These possibility offered by Wavelet Transformed is used to analyze and characterize the conductivity of the sciatic nerve, object of this work.

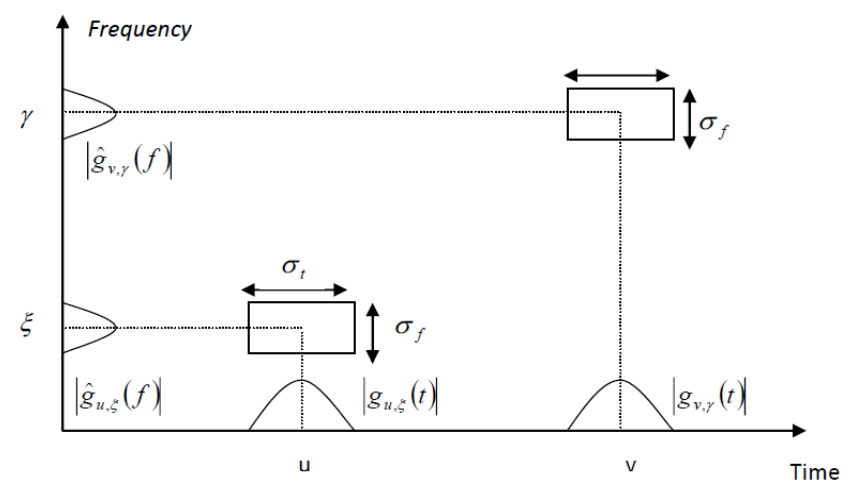

Figure 2: Time-frequency energy of Gabor atom. 


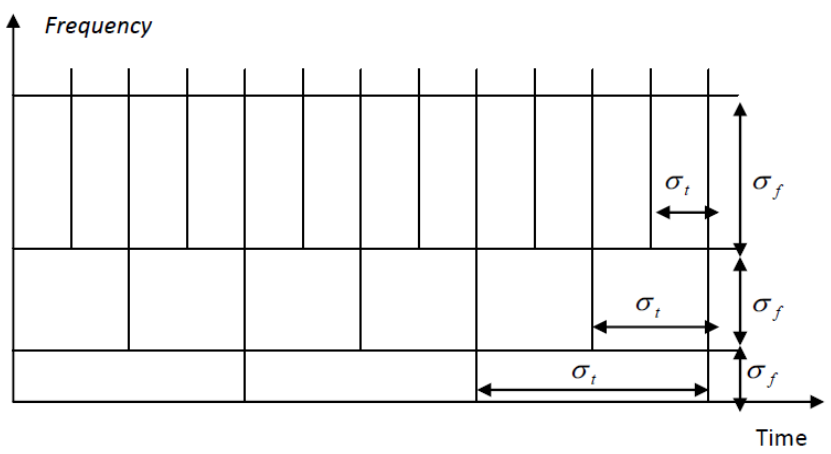

Figure 3: Time-frequency division using wavelet basis function.

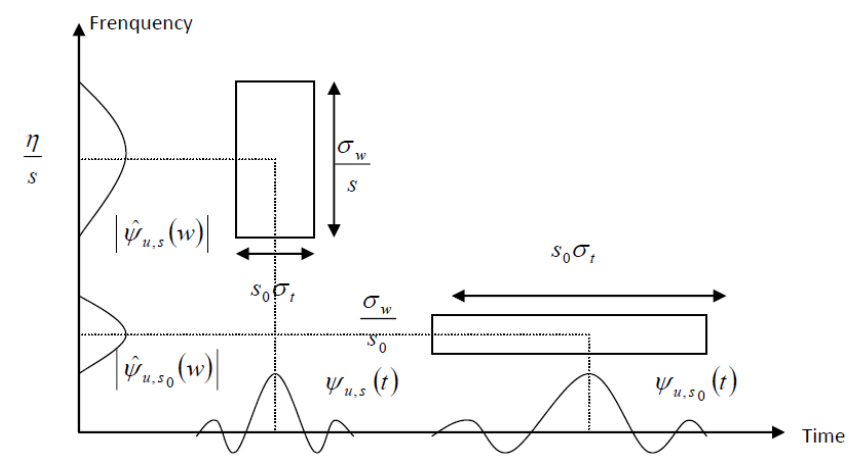

Figure 4: Time-frequency energy of wavelet function.

\section{Material and Method}

\section{Analysis and characterization of the conductivity behaviour of the sciatic nerve}

Sciatic nerve samples $(\mathrm{n}=6)$ were obtained by decapitation of frogs (Rana esculenta) with light anesthesia (Halothane $2.5 \%$ in air). The proximal segments of the sciatic nerves $(1 \mathrm{~cm})$ were harvested in order to study resistivity. Animals were cared for, under the Tunisian code of practice for the Care and Use of Animals for Scientific Purposes. The experimental protocols were approved by the Faculty Ethics Committee (Faculté des Sciences de Bizerte, Tunisia). Sciatic nerves were conserved in Ringer-buffer during 1 to $5 \mathrm{~min}$.

Then, the electrical resistivity variations of the sciatic nerve with varying temperature were investigated by employing the four-probe technique. The temperature variation was achieved using a Helium exchange gas filled cryostat (closed cycle refrigerator). Temperature was measured using a calibrated Si-diode sensor with an accuracy of $0.1{ }^{\circ} \mathrm{K}$ and was varied from $300^{\circ} \mathrm{K}$ to $20^{\circ} \mathrm{K}$. In the present experiment, we study the effect of the decrease and the increase of temperature on nerve resistivity. The two external wires (the distance between the 'current' wires: $8 \mathrm{~mm}$ ) were used as current leads and the other two as the voltage leads (the distance between the 'current' wires: $2 \mathrm{~mm}$ ) to record potential differences. We used a variable current with very low frequency $(36 \mathrm{~Hz})$. The value of the current used for the resistivity measurements was $20 \mu \mathrm{A}$ (micro Ampere) [3,5]. The four-point probe technique is the most common method of determining the critical temperature $(\mathrm{Tc})$ of a superconductor. $\mathrm{T}^{\text {conset }}$ is the temperature at which resistivity starts decreasing. Wires are attached to a material. Through two of these points, a voltage is applied and if the material is conductive, a current will flow. Then, if any resistance exists in the material, a voltage will appear across the other two points in accordance with the Ohm's law. When the material enters a superconductor state, its resistance drops to zero and no voltage appears across the second set of points. The Figure 5 shows the Four-point technique used for measuring the resistivity of the sciatic nerve.

\section{Analysis of the electrical conductivity of sciatic nerve}

To analysis the regularity of a function (in our case the function is a signal), the continuous wavelet transform is desired versus discrete wavelet transform. Singularity detection can be undertaken by describing the local regularity of the signal. In our approach, we take advantage of the ability of the wavelet transform to characterize the local regularity of sciatic nerve signal. The mathematical background justifying this method is described in Mallat and Hwang [6-8] USA. For a given signal, the local regularity consists to compute all modulus maxima of its continuous wavelet transform and chain maxima across scales to obtain maxima lines. If the signal is noisy, maxima lines due to noise are mostly concentrated at fine scales, whereas maxima lines due to signal discontinuity should be persistent across coarser scales. Mathematically, singularity detection can be carried out by finding the abscissa where the wavelet modulus maxima converge at finer scales. If no wavelet modulus maxima exists at fine scales in a point for which the abscissa is $t=u$, it was shown that the signal is regular at this point. The major difficulty is to distinguish singularities caused by noise fluctuations from those that generated from sharp signal transitions. Only maxima lines that persist across all scales of the analysis are considered as true signal transitions, since noise fluctuations should have less persistence in scale-space. The task is to characterize the regularity of singularities through their Lipscitz exponent $\alpha$. In general, the point-wise regularity of a function can be characterised by Lipschitz exponents. Mallat and Hwang [6] showed that Lipschitz exponents could be estimated from the decay of wavelet modulus maxima defined in the equation 11 :

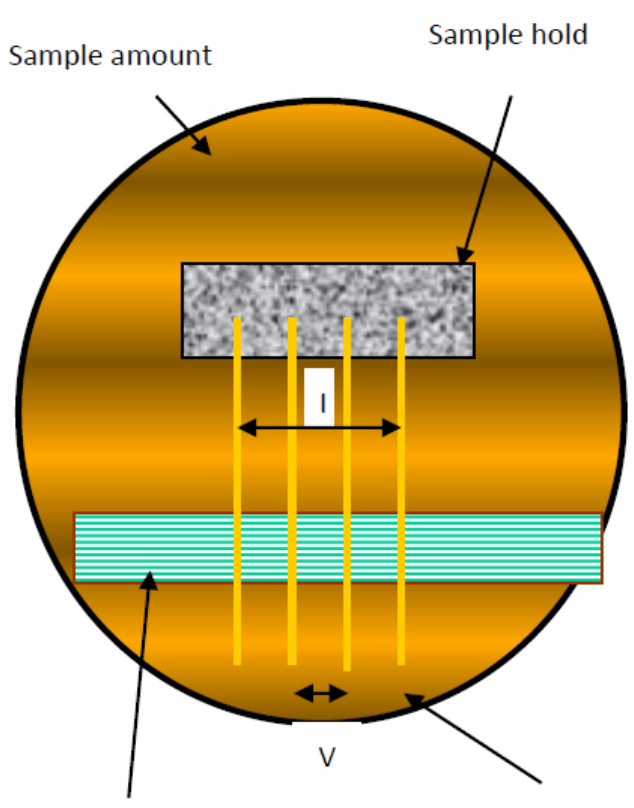

Sample wiring

Sciatic nerve

Figure 5: The four-point probe technique for nerve conductive studies. 


$$
|W f(u, s)| \leq A s^{\alpha+\frac{1}{2}}
$$

Where $A$ is a constant, $s$ the scale parameter, and $u$ is the translation parameter. The modulus of the wavelet coefficients varies with the scale according to the Lipschitz regularity $\alpha$. The equation 4 is equivalent to the equation 12:

$$
\log _{2}(|W f(u, s)|) \leq \log _{2}(A)+\left(\alpha+\frac{1}{2}\right) \log _{2}(s)
$$

Hence, the Lipschitz regularity can be estimated from the slope of $\log _{2}(|W f(u, s)|)$ as a function of $\log _{2}(s)$ along the maxima lines. We can estimate Lipschitz $\alpha$ from the decay slope of $\log _{2}(|W f(u, s)|)$. Note that a step edge is Lipschitz 0 . The wavelets used in this work have one and two vanishing moments. Essentially, singularities with more negative exponents should be attributed to noise, whereas signal transitions should have more positive exponents [7].

So, given a signal with several localized discontinuities, these localized discontinuities may be located and characterized by the estimation of $\alpha$ using the maxima wavelet coefficients called Wavelet Transform Modulus Maxima (WTMM). The measuring of the slope of the WTMM curve allows the estimating of $\alpha$ which is the characteristic of the singularity. The choice of wavelet basis depends on the application and in this work we have chosen the Gaussian Continuous Wavelet (GCW) which has the property to conserve the regularity of signal at different scales.

\section{Experimental Results}

The Figures 6 and 7 show the responses of the frog sciatic nerve versus temperature in Kelvin degree. In the Figure 6, the measure equipment is placed outside the sciatic nerve and in the Figure 7, the measure equipment is placed inside the sciatic nerve. These responses are the ratio of $\mathrm{R}$ by $\mathrm{Ra}$ where $\mathrm{Ra}$ is the nerve resistance at the room temperature $\left(298{ }^{\circ} \mathrm{K}\right)$, and $\mathrm{R}$ is the nerve resistance at different values of the temperature. The curves shown in the Figures 6 and 7 may be considered as the impulse response of the sciatic nerve and describe the conductivity behaviour of frog sciatic nerve at different values of the temperature.

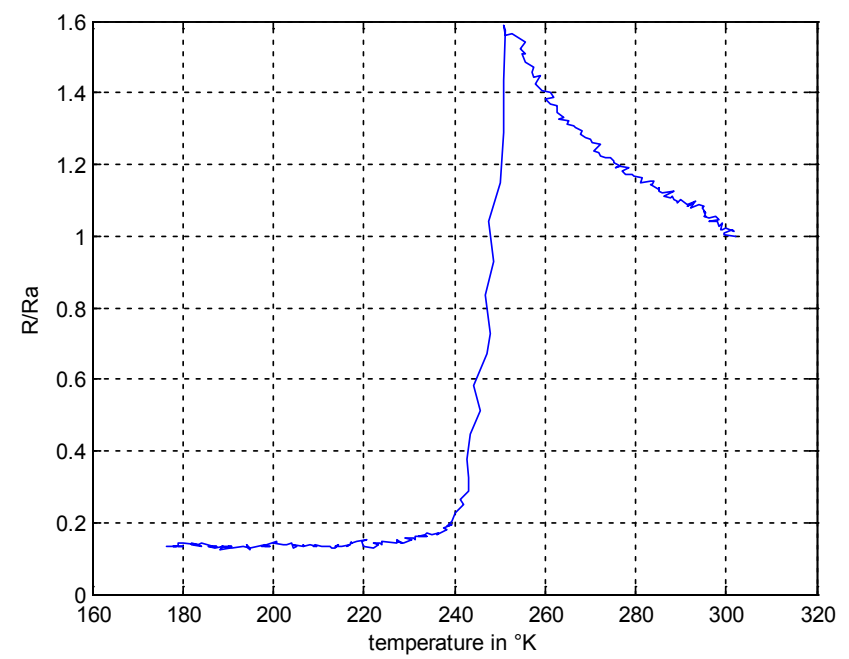

Figure 6: Impulse response $(\mathrm{R} / \mathrm{Ra})$ of nerve versus temperature in Kelvin degree: the measure equipment is placed outside the sciatic nerve.

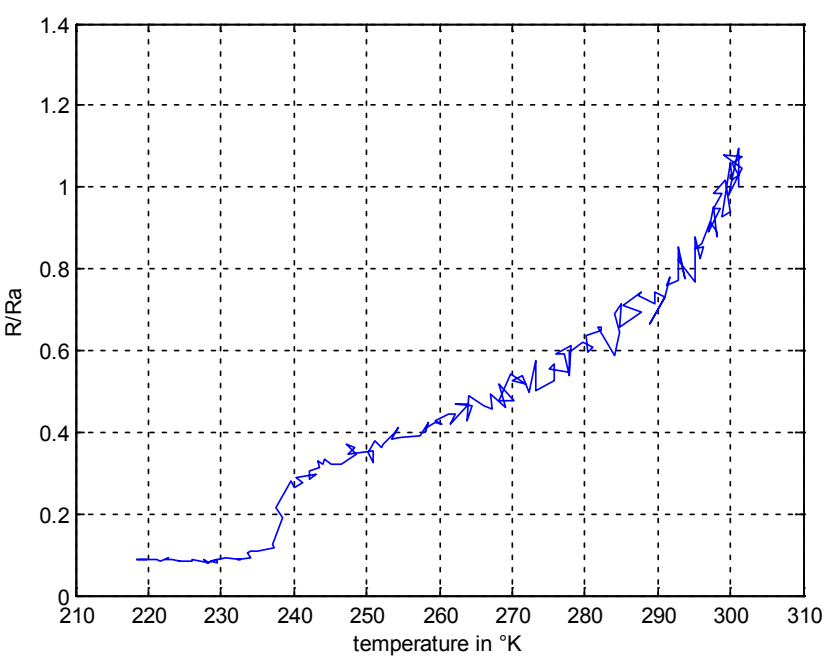

Figure 7: Impulse response (R/Ra) of nerve versus temperature in Kelvin degree: the measure equipment is placed inside the sciatic nerve.
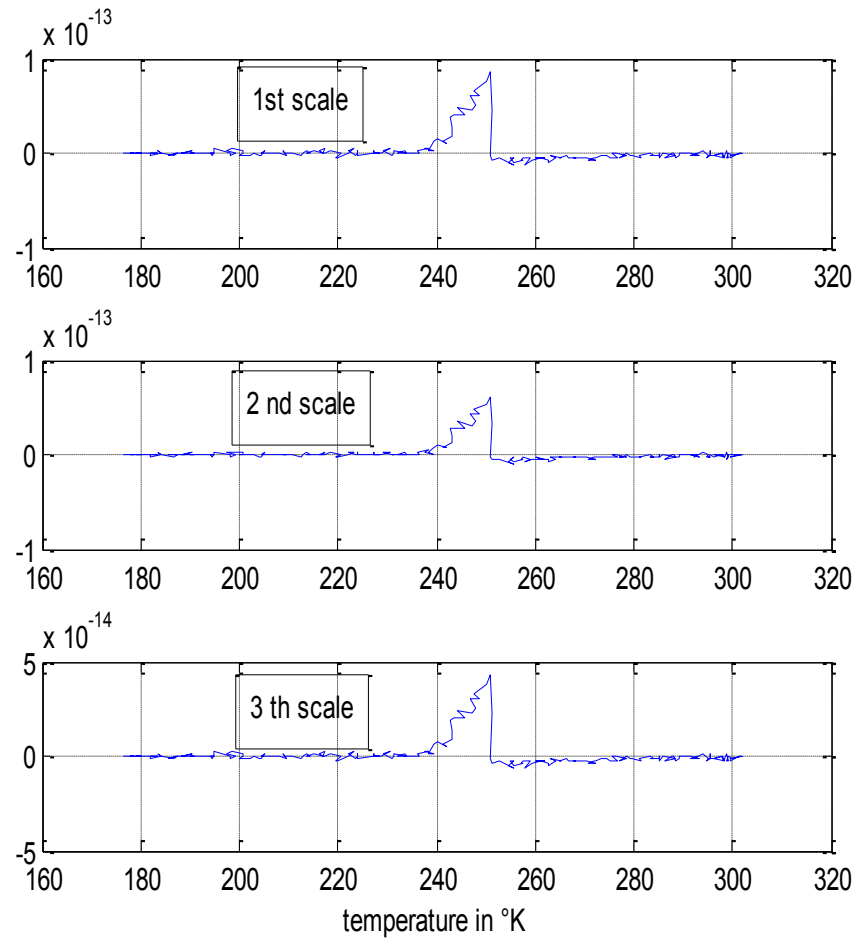

Figure 8: Wavelet coefficients representation for $1^{\text {st }}, 2^{\text {nd }}$ and $3^{\text {th }}$ scales versus temperature in Kelvin degree: the measure equipment is placed outside the sciatic nerve.

We decompose the signal of the Figures 6 and 7 by the continuous wavelet using GCW. Five scales are performed with GCW. The Figures 8-11 show the wavelet representation for five scales. The Figure 8 shows the wavelet coefficients of the frog sciatic nerve for the first, the second and the third scales, the Figure 9 shows the wavelet coefficients of the frog sciatic nerve for the fourth and fifth scales, both figures for the measure equipment placed outside the sciatic nerve. The Figure 10 shows the wavelet coefficients of the frog sciatic nerve for the first, the second and the third scales, the Figure 11 shows the wavelet coefficients of the frog sciatic nerve for the fourth and fifth scales, both figures for the measure equipment placed inside the sciatic nerve (Figure 6). 

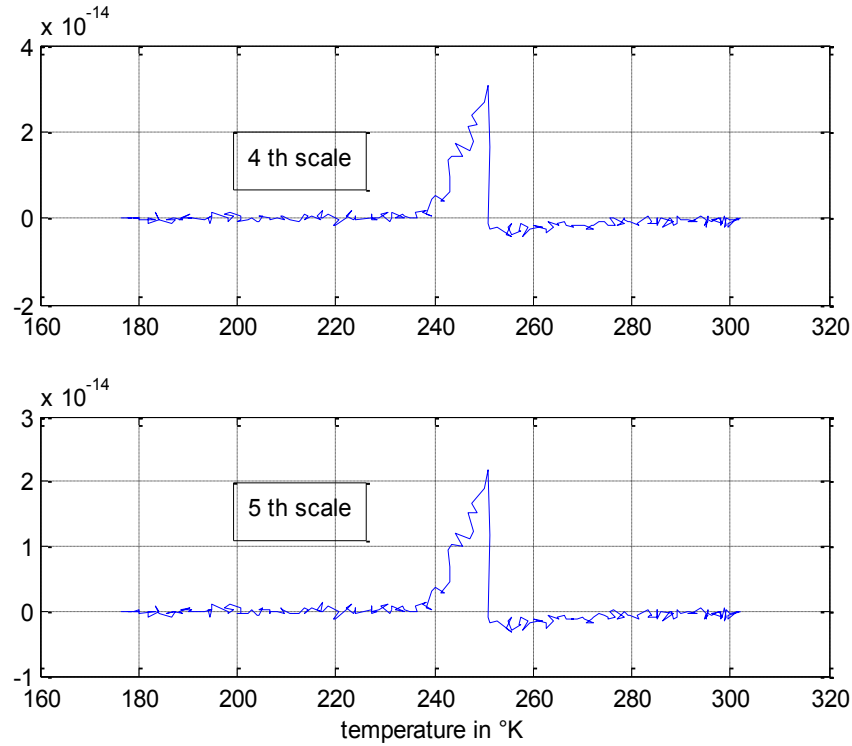

Figure 9: Wavelet coefficients representation for $4^{\text {th }}$ and $5^{\text {th }}$ scales versus temperature in Kelvin degree: the measure equipment is placed outside the sciatic nerve.
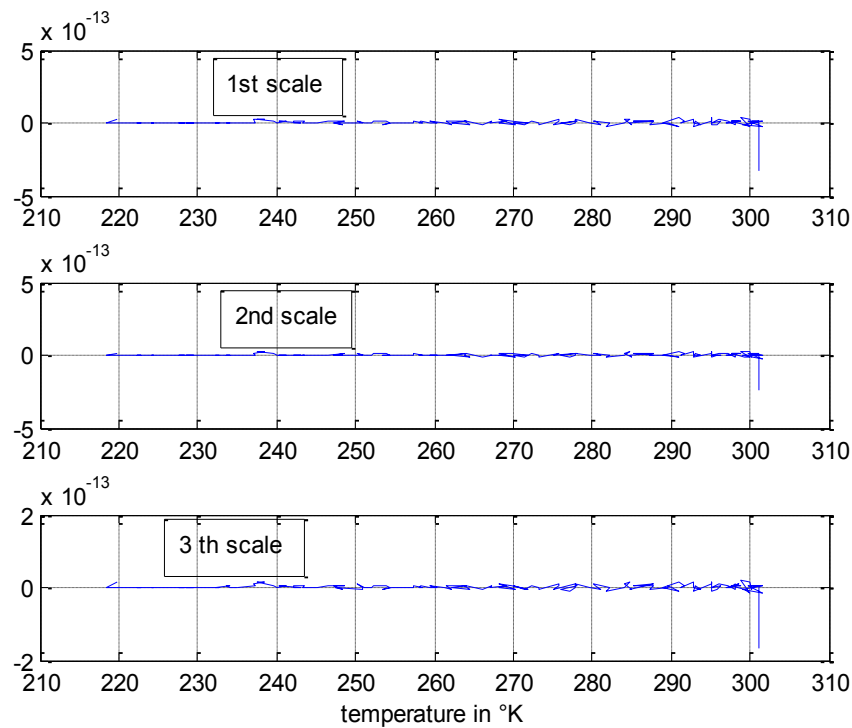

Figure 10: Wavelet coefficients representation for $1^{\text {st }}, 2^{\text {nd }}$ and $3^{\text {th }}$ scales versus temperature in Kelvin degree: the measure equipment is placed inside the sciatic nerve.

The observation of the Figures 8-11 shows that the transition in the response of the nerve is well represented in the wavelet domain particularly for the Figure 8 and 9 where the measure equipment is placed outside the sciatic nerve. The Figures 12 and 13 are the scalespace representation of the response which may be considered as an image representation of the sciatic nerve response; the transitions in the nerve response are also well observed in these images, particularly in Figure 12.

The transition observed in Figures 8-11 may be considered, in signal processing point of view, as the singularity behaviour and our goal is to determine the type of this singularity. First, we detect the local maxima of the wavelet coefficients for each scale (we have five
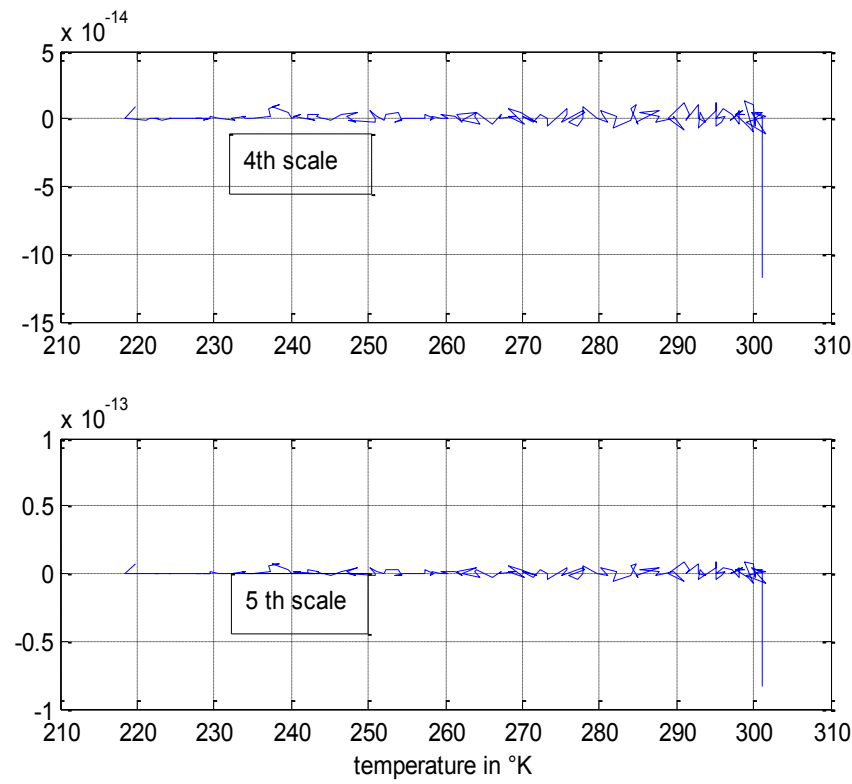

Figure 11: Wavelet coefficients representation for $3^{\text {th }} 4^{\text {th }}$ and $5^{\text {th }}$ scales versus temperature in Kelvin degree: the measure equipment is placed inside the sciatic nerve.

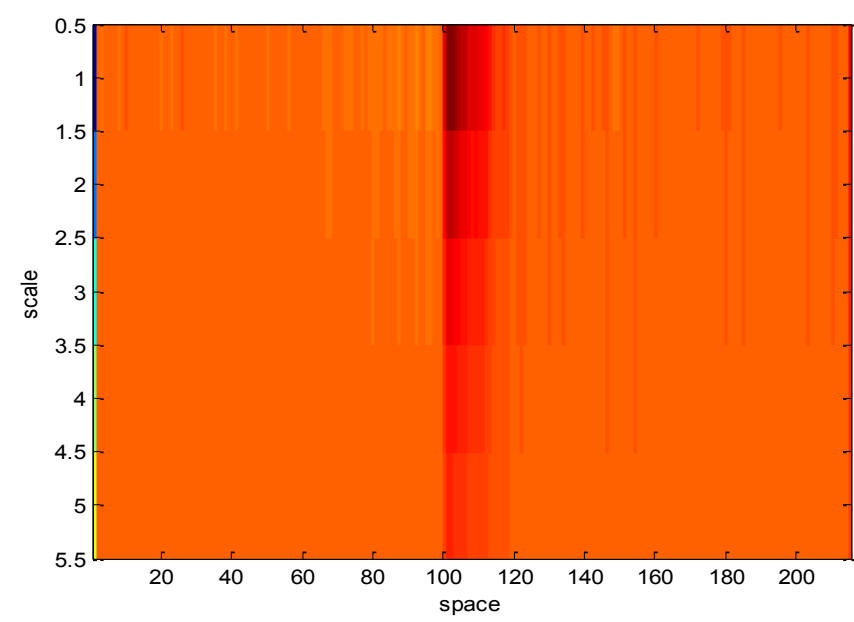

Figure 12: Image representation in the scale-space domain of the sciatic nerve response: the measure equipment is placed outside the sciatic nerve.

scales in our decomposition). Second, we calculate the logarithm of the absolute value of these local maxima. Third, we calculate the logarithm of the scale vector containing the five scales. Fourth, we represent the logarithm of the absolute values versus the logarithm of the scale vector. The Figures 13-15 show the results obtained. The Figure 14 shows the curve for the measure equipment placed outside the sciatic nerve; the Figure 15 shows the curve for the measure equipment placed inside the sciatic nerve Mbainaibeye et al. [8].

These curves are a graphical representation of a linear function and may be described by the equation 13 :

$$
y=a x
$$

Where $y$ is the logarithm of absolute values of the maxima of wavelet coefficients, $x$ is the logarithm of scale vector, $a$ is the slope of the curve Mbainaibeye et al. [8]. 


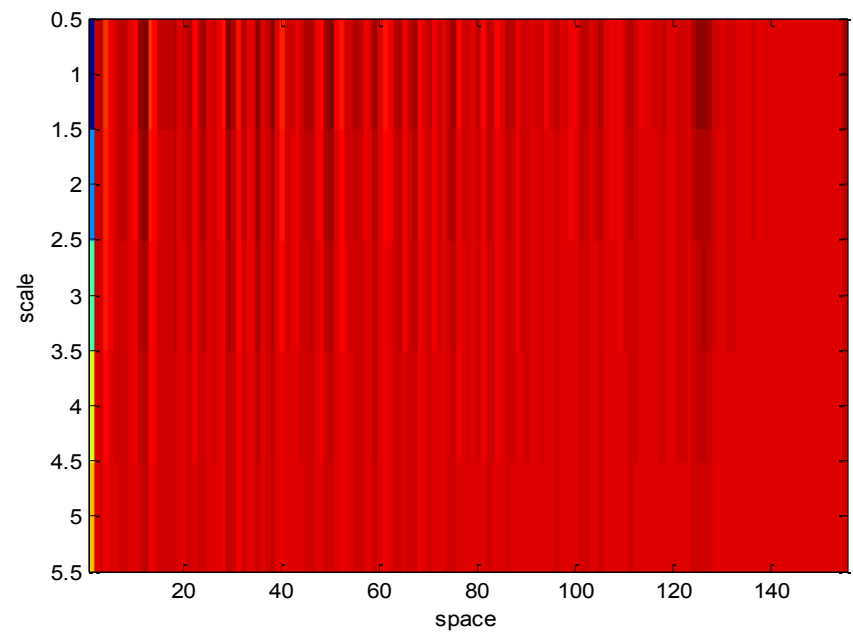

Figure 13: Image representation in the scale-space domain of the sciatic nerve response: the measure equipment is placed inside the sciatic nerve.

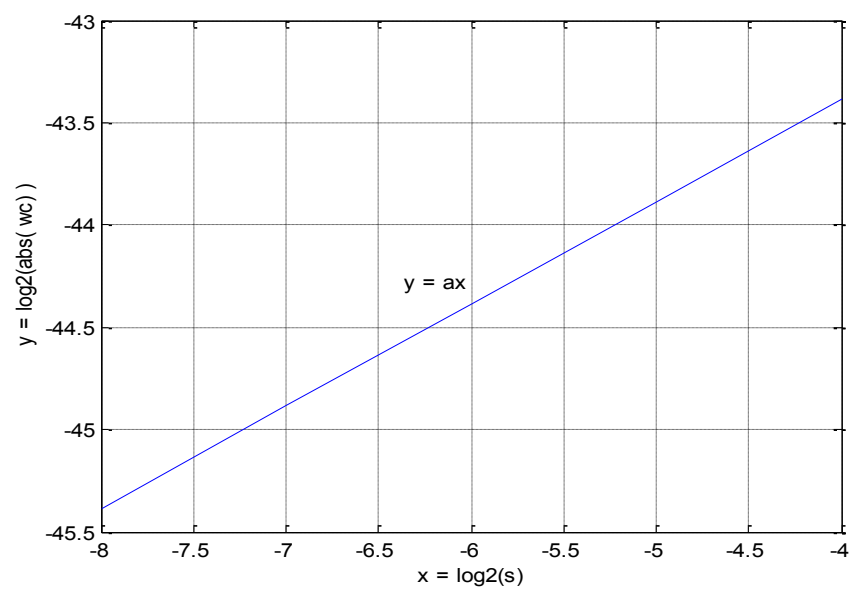

Figure 14: Curve of local maxima versus scale: the measure equipment is placed outside the sciatic nerve.

Since $y$ is a linear function of $x$, the value of $a$ may be determined by the equation 14 :

$$
a=\frac{y(k)-y(j)}{x(k)-x(j)}
$$

Where $\mathrm{k}$ and $j$ are the any index of the function. The Figures 16 and 17 show the results of the equations 13 and 14:

The transition of the sciatic behaviour is particularly pronounced in the Figure 6, transition observed by placing the measure equipment outside the sciatic nerve. We quantitatively evaluate the sensibility of this transition between $250^{\circ} \mathrm{K}$ and $240{ }^{\circ} \mathrm{K}$ in term of variations of the ratio $\mathrm{R} / \mathrm{Ra}$ by Kelvin degree in the zone of transition. We design by $\mathrm{S}_{\mathrm{K}}$ this sensibility and $\mathrm{V}$ the value of the response of the sciatic nerve at the temperature $\mathrm{T}$ in ${ }^{\circ} \mathrm{K}$. Since the impulse response shown in the Figure 6 is a ratio of $\mathrm{R}$ by $\mathrm{Ra}, \mathrm{S}_{\mathrm{K}}$ may be estimated by the equation 15 :

$$
S_{K}=\frac{V(240)-V(250)}{240-250}=\frac{0.2-1.6}{240-250}=0.14 \mathrm{~K}^{-1}
$$

So, this value indicates that between the semi-conductor and the supra-conductor behaviour of the frog sciatic nerve, the sensibility in term of $\mathrm{R}$ by $\mathrm{Ra}$ ration is 0.14 per $^{\circ} \mathrm{K}$ (Figure 18) [8].

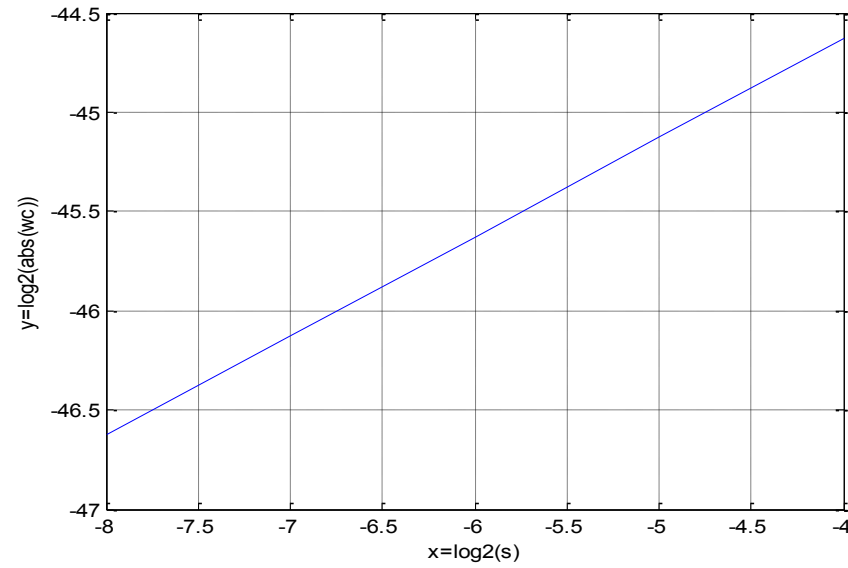

Figure 15: Curve of local maxima versus scale: the measure equipment is placed inside the sciatic nerve.

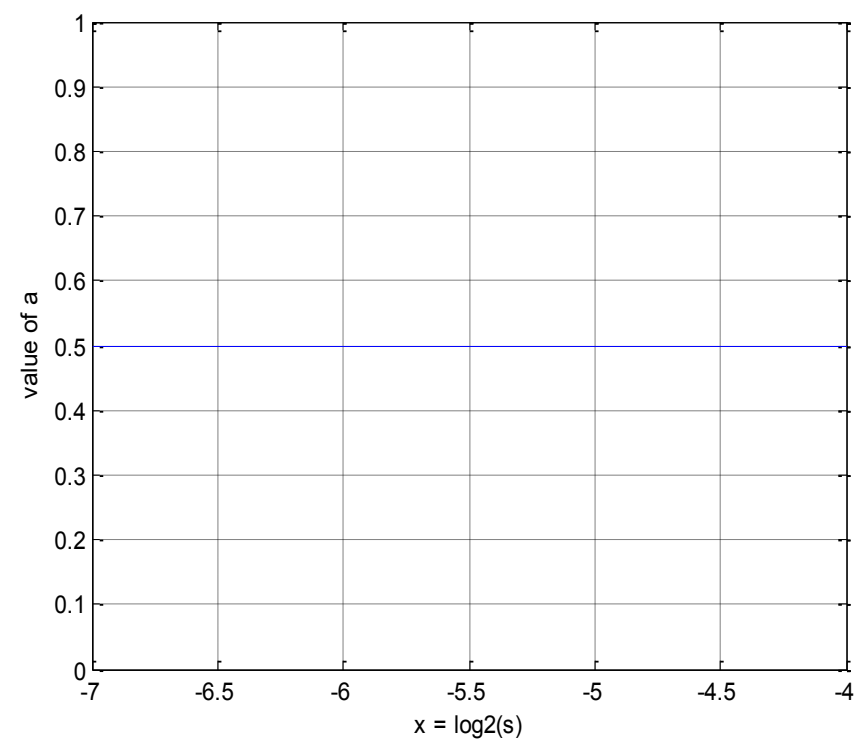

Figure 16: Value of aversus scale: the measure equipment is placed outside the sciatic nerve.

\section{Discussion}

The present investigation point to the modulation of neurochemical, electric properties, metabolic rate by cold and many hormone like glucagon. Moreover, data indicated that low temperatures induce a fall of nerve resistivity in frog; showing a superconductor-like behaviour. Previous results have demonstrated the existence of superconductor-like behaviour in sciatic nerve $[3,5]$. Our main interest lies in the electrical properties of large-scale nerve networks at low temperature during physiological or pathological situations. Understanding these complex functions of nerves during different sychological status requires a multidisciplinary approach [1-5] reported that superconductor-like behaviour is related to the development of metabolic function, the development of myelin and the three-dimensional arrangement of proteins and ion channels. Nerve fibres conduct nerve impulses very quickly because the myelin sheath has gaps, which allows the nerve impulse to jump from gap to gap and travel faster [3]. The mechanism of superconductivity and the saltation over the myelin sheathed portions of the nerves showed many similarities with superconductors. Interestingly, in superconductor 


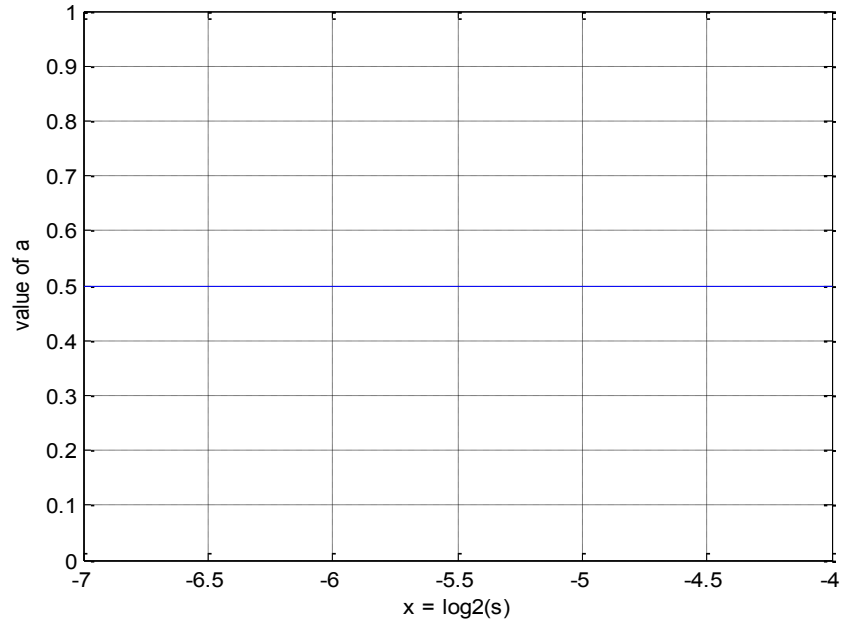

Figure 17: Value of aversus scale: the measure equipment is placed inside the sciatic nerve.

materials we found Josephson junction. Josephson Junctions are thin layer of insulating material sandwiched between two superconducting layers. Electrons "Tunnel" through this non-superconducting region in what is known as the Josephson effects Mbainaibeye et al. [8]. Here a superconducting current flows even in the absence of an external voltage. Moreover, the gradient shift of superconductor-like behaviour in the nervous system from poikilotherms to endotherms is correlated with the degree of myelinisation and one exemple of energy management [3].

Energy could be understood as conventional measurement of metabolic rate with thermochemistry approches based on QR indicat the metabolism nature (glucidic, lipidic, or protidic) or innovative approach to understand the energy management in the brain or nervous system like superconductor-behaviour that explain the improvement of information treatment and electric conduction without heat loss. Moreover, we can discuss the energy fluctuation as dynamic energy (Figure 18) on the body. The dynamic energy move through the body (digestive tube, etc) and vary with the body shape in female and male. The electromagnetic pollution (artificial EMF) could alter the equilibrum of the dynamic energy and metabolic rate (metabolic energy) in order to explain stress, diabete, hypoxia, cancer...etc and behaviour or neurological disease like amnesia, anxiety, or Alzheimer $[9,10]$.

We have to consider three level of energy in the body (E0 physiological, E1 : high energy, E3 : low energy) (Figure 18). Energy level is represented on a scale of -1 (lowest) and 1 (highest). Energy fluctuation of between negative or positive is related to body shape (man or women), metabolic rate, and environment (pollution, electromagnetic field pollution, stress,...etc). When nervous system works as superconductor-like behavior we have E0 energy and its fluctuation lead to metabolic disorder or modulate positively or negatively our emotions. We can draw three circles of energy (body energy, nervous system energy, environment energy) four different behavior that represent energy states as high or low and as either positive or negative. Low energy fluctuation from E0 (physiological for the body and nervous system) and weak noise in the environment explain good health. The energy fluctuation in one circle (E1 or E-1) lead to different behaviour and emotions implicated in the disease of genesis. Interestingly, alteration of superconductor-behaviour could be associated with neurodegenerative disease or differtens motor

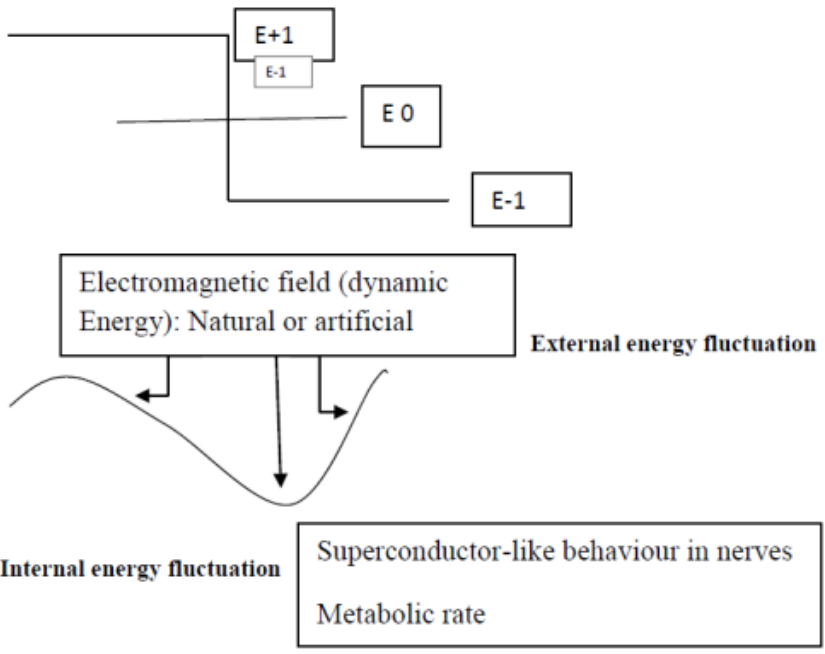

Figure 18: Body shape and Energy fluctuation (E-1, E O, E+1).

dysfunction as paralysia in Human and animal. The diseases associated to the alteration of superconductor-like behavior could be considered as an increase of heat loss and energy with low information treatment.

Harvesting natural energy (solar electromagnetic field) or artificial (electromagnetic field generated from mobile phone, WIFI, WIMAX...) in the Human or animal environment has been identified as an effective way to modulate the body energy in wireless body area networks such as superconductor-like behaviour in nervous sytem (NS) based on the EMF circulation through myelin nanopares or mitochondria in cells. Morover, eating heavily could influence energy balance with classical physiology based on respiratory thermochemistry (QR) associated to digestive wastes in the body that can be linked to the possible interaction of electromagneticfield with fuel metabolite in the digestive tube. Natural and artificial electromagnetic field can act on the body surface and modulate body energy positively or negatively with different mechanisms based on size and body shape in Human and animals.

\section{Conclusion}

The analysis by the wavelet transform shows that between 250 ${ }^{\circ} \mathrm{K}$ and $240{ }^{\circ} \mathrm{K}$, the frog sciatic nerve presents a 0.5 singularity order which indicates the magnitude discontinuity in its electrical resistance behaviour. The main contribution of this review is that we report the existence of superconductor-like behaviour in sciatic nerves and the precise characterization by the wavelet transform of the impulse response of the sciatic nerve.

In addition, we introduce a theory related to energy management between three circles (Body energy, nervous sytem energy, and environment energy) that explain in part health or diseases in Human or animals.

\section{References}

1. Abdelmelek H, Cottet-Emard JM, Pequignot JM, Barré H (2000) Spinal cord monoaminergic system response to age and cold-acclimatization in muscovy duckling. J Neural Transm 107: 1175-1185.

2. Abdelmelek H, Fechtali T, Rouanet JL, Montaron A, Lachuer J, et al. (2001) Effects of intracerebroventricular injections of des-His1 (Glu9) glucagon amide on the regulatory thermogenesis in Muscovy ducklings. C R Acad Sci III 323: 267-271.

3. Abdelmelek H, Chirgui MA, Salem BM, Sakly M (2003) Impact of evolution on the electrical properties of sciatic nerves: Superconductivity-like. Phys Chem 13: $132-134$. 
Citation: Abdelmelek H, Hanini A, Saili L, Azzouz A, Nebil E, et al. (2017) Energy Management and Health: Implication of Nervous System Superconductor-Like Behaviour. J Health Med Informat 8: 289. doi: 10.4172/2157-7420.1000289

Page 8 of 8

4. Abdelmelek H, El-May B, Hamouda A, Ben Salem M, Pequignot JM, et al. (2011) Electrical conduction through nerve and DNA. Prog Biophys Mol Biol 46: 1-8.

5. Abdelmelek H, Cottet-Emard JM, Pequignot JM, Barré H (2003) Sciatic nerve monoaminergic system responses to cold acclimatization in Muscovy duckling. J Neural Transm 110: 1359-1367.

6. Mallat S, Hwang WL (1992) Singularity detection and processing with wavelets. IEEE 38: 617-643.

7. Mallat S (1998) A Wavelet Tour of Signal Processing 1998: 1-123.
8. Bouyahia SM, Mbainaibeye J, Ellouze N (2012) Characterization of singularities by wavelet transform modulus maxima: Application to Microcalcifications detection in digitized mammogram.

9. Othman H, Ammari M, Rtibi K, Bensaid N, Sakly M, et al. (2017) Postnatal development and behavior effects of in-utero exposure of rats to radiofrequency waves emitted from conventional WiFi devices. Environ Toxicol Pharmacol 52: 239-247.

10. Saili L, Hanini A, Smirani C, Azzouz I, Azzouz A, et al. (2015) Effects of acute exposure to WIFI signals $(2.45 \mathrm{GHz})$ on heart variability and blood pressure in Albinos rabbit. Environ Toxicol Pharmacol 40: 600-605. 